\title{
Ultra-Low-Dose CT: An Effective Follow-Up Imaging Modality for Ureterolithiasis
}

\author{
Robin Z. Cheng, BS, Eugene Shkolyar, MD, ${ }^{1}$ Tim C. Chang, MD, ${ }^{1}$ Kyle Spradling, MD, ${ }^{1}$ Calyani Ganesan, MD, \\ Shen Song, MD, ${ }^{2}$ Alan C. Pao, MD, ${ }^{2}$ John T. Leppert, MD, MS, ${ }^{1}$ Christopher S. Elliott, MD, PhD, ${ }^{3}$ \\ Katherine To'o, MD, ${ }^{4}$ and Simon L. Conti, MD, MEd ${ }^{1}$
}

\begin{abstract}
Background and Purpose: Classically, abdominal X-ray (KUB), ultrasound, or a combination of both have been routinely used for ureteral stone surveillance after initial diagnosis. More recently, ultra-low-dose CT (ULD CT) has emerged as a CT technique that reduces radiation dose while maintaining high sensitivity and specificity for urinary stone detection. We aim to evaluate our initial experience with ULD CT for patients with ureterolithiasis, measuring real-world radiation doses and stone detection performance.

Methods: We reviewed all ULD CT scans performed at the Veterans Affairs Palo Alto Health Care System between 2016 and 2018. We included patients with ureteral stones and calculated the mean effective radiation dose per scan. We determined stone location and size, if the stone was visible on the associated KUB or CT scout film, and if hydronephrosis was present. We performed logistic regression to identify variables associated with visibility on KUB or CT scout film and hydronephrosis.

Results: One hundred eighteen ULD scans were reviewed, of which 50 detected ureteral stones. The mean effective radiation dose was $1.04 \pm 0.41 \mathrm{mSv}$. Of the ULD CTs that detected ureterolithiasis, $38 \%$ lacked visibility on KUB/CT scout film and had no associated hydronephrosis, suggesting that they would be missed with a combination of KUB and ultrasound. Larger stones (odds ratio [OR]: 1.40, 95\% confidence interval [CI]: $1.08,1.96$ for every $1 \mathrm{~mm}$ increase in stone size) were more likely to be detected by KUB/CT scout film or ultrasound, while stones in the distal ureter (OR: $0.18,95 \%$ CI: $0.03,0.81$ ) were more likely to be missed by KUB/CT scout film or hydronephrosis.

Conclusions: Based on our institutions' initial experience, ULD CT detects small and distal ureteral stones that would likely be missed by KUB or ultrasound, while maintaining a low effective radiation dose. An ULD CT protocol should be considered when reimaging for ureteral stones is necessary.
\end{abstract}

Keywords: kidney stone, CT, ultra-low dose

\section{Introduction}

$\mathbf{U}$ RINARY STONE DISEASE AFFECTS $8.8 \%$ of the U.S. population. ${ }^{1}$ Upper urinary tract stones account for 1.5 million emergency room visits every year, with annual health care costs totaling up to $\$ 5.3$ billion. ${ }^{2-4}$ Noncontrast CT scans of the abdomen and pelvis provide excellent sensitivity and specificity for stone detection, and are considered the gold standard test for diagnosing ureterolithiasis. ${ }^{5}$ In addition, noncontrast CT provides the most accurate data on stone location and size. ${ }^{5,6}$ Other common imaging modalities such as plain abdominal X-ray (KUB) suffer from poor sensitivity $(50 \%)$ and specificity $(76 \%)$, while ultrasound is inferior to
CT in stone detection (sensitivity $72 \%$, specificity $73 \%$ ) and sizing. 5,7 Ultrasound usually does not directly visualize middle to distal ureteral stones but rather only suggests their presence through a finding of hydronephrosis.

Standard CT Imaging of upper urinary tract stones requires higher doses of ionizing radiation. Recent efforts have focused on CT dose modification in an effort to prevent unwanted long-term health effects (e.g., cancer). ${ }^{8,9}$ Low-dose scans have been shown to have $59 \%$ lower organ-specific radiation compared with standard CT with no loss of image quality. ${ }^{5,10}$ More recently, ultra-low-dose CT (ULD CT) protocols, which use even less ionizing radiation than lowdose CT, have been developed. A systematic review

Departments of ${ }^{1}$ Urology and ${ }^{2}$ Nephrology, Stanford University School of Medicine, Stanford, California.

${ }^{3}$ Department of Urology, Santa Clara Valley Medical Center, San Jose, California.

${ }^{4}$ Department of Radiology, Stanford University School of Medicine, Stanford, California. 
demonstrated that ULD CT $(<1.9 \mathrm{mSv})$ and low-dose CT $(<3.5 \mathrm{mSv})$ have comparable sensitivity $(90 \%-96 \%)$ and specificity $(97 \%-99 \%)$ for ureteral stones. ${ }^{11-13}$

Standard CT imaging protocols are often used to diagnose upper tract urinary stones, while KUB and ultrasound are the conventional imaging modalities for follow-up of stone passage during a trial of medical expulsive therapy. ${ }^{5}$ There is no established imaging protocol in following up the $36 \%$ of cases where stone symptoms dissipate after 4 weeks of medical expulsive therapy, but it is unclear if the stone has passed. ${ }^{14,15}$ In the absence of indicative symptoms or visual confirmation of passage, imaging is necessary to track stone progression or resolution as silent obstruction can lead to renal damage. ${ }^{16}$ Our aim was to provide data on the radiation exposure from ULD CT, as well as to assess the added utility of ULD CT over KUB and ultrasound in detecting ureteral stones.

\section{Methods}

\section{ULD CT protocol}

After obtaining an exemption from our institutional review board we performed a retrospective study of patients undergoing ULD CT from August 2016 to February 2019 at the Veterans Affairs Palo Alto Health Care System with confirmed or suspected ureteral stones.

We defined an ULD CT as a study with dose equivalent of $<1.9 \mathrm{mSv},{ }^{11}$ which is lower than the mean effective dose used to perform abdominal film KUBs $(\sim 2.15 \mathrm{mSv})$, which require multiple views to capture the abdomen and pelvis. ${ }^{17}$ To be eligible for ULD CT at our institution a patient was required to have a BMI $<40$. This exclusion criterion is based on the finding that low-dose CT has low sensitivity and specificity for ureteral stones in patients with $\mathrm{BMI} \geq 30$, and thus is not recommended in this population. ${ }^{18}$ In addition, due to the limitations of ULD CT imaging on solid abdominal organs and the concern for false positives not related to renal or ureteral stones, a prior low-dose or standard-dose CT was required within 18 months before the ULD CT.

We performed all ULD CT scans without contrast on a 64section CT scanner (Discovery CT750 HD; GE Healthcare, Milwaukee, WI) by using automated tube current modulation (SmartmA; GE Healthcare), 0.5-seconds rotation time, and a pitch of 1.375 . We restricted the scan coverage area to the urinary tract, from the top of the kidneys to the pubic symphysis. We set the range of the tube current for low-BMI protocol to $75-150 \mathrm{~mA}$, the tube voltage to $80 \mathrm{kV}$, and noise index to 25 . The range of tube current for high $\mathrm{BMI} \geq 30$ but $\leq 40$ was set to $75-150 \mathrm{~mA}$; the tube voltage was set to $80 \mathrm{kV}$, noise index to 30. Examinations were reconstructed with a standard kernel for filter back projection and with adaptive statistical iterative reconstruction (ASIR; GE Healthcare) using a ratio of $70 \%$. The noise index and ASIR level selected were based on our experience with the use of ASIR for various indications in the abdomen as well as general agreement about image-quality expectations among the subspecialty radiologists. Images were reformatted in the coronal and sagittal planes by using a $2.5 \mathrm{~mm}$ section thickness on the scanner console immediately after completion of the CT examinations. All the image data sets were then transmitted to the picture archiving and communication system for image interpretation.

\section{Data collection}

We abstracted patient demographics, medical history, and laboratory data from review of the electronic medical record. We reviewed the final interpretation of ULD CT by the reading radiologist and information regarding presence or absence of stones, stone size and location, and presence of hydronephrosis. A urologist (S.L.C.) reviewed each scan to corroborate the radiology read and examine the CT scout film to determine if a ureteral stone might be detected by a KUB. If formal KUB had been obtained as part of the work-up, we used the KUB in lieu of the CT scout film. Ureteral stones seen on CT scout film were used as a surrogate for visibility of ureteral stones on KUB when patients who did not have associated KUB taken. In the subset of patients who had KUBs available, we tested if ureteral stone detection differed significantly between KUB and ULD CT scout film using Fisher's exact test. We used the presence of hydronephrosis on the ULD CT as a surrogate for visibility of hydronephrosis on ultrasound to determine the approximate sensitivity of ultrasound to detect ureteral stones.

We fit univariable and multivariable logistic regression models to determine features associated with stone detection by KUB/CT scout film or hydronephrosis. We performed statistical analysis using Rstudio software (Rstudio Team, $2016^{19}$ ) and considered two-way $p$-values of 0.05 as our statistical significance threshold.

\section{Results}

Of the 118 ULD CT scans performed, 50 (42.4\%) detected ureteral stones (Table 1). Of the 50 ureteral stones, $92.0 \%$ were $\leq 9 \mathrm{~mm}$ with an average stone size of $5.96 \pm 2.85 \mathrm{~mm}$ (Fig. 1). Of those with ureteral stones, 15 ULD CT scans (30.0\%) did not have corresponding KUBs, and a CT scout film was used as a proxy. Visibility of ureteral stones by several imaging criteria was assessed (Table 2). By location,

Table 1. Baseline Characteristics for Patient Cases With Ureteral Stones ON Ultra-Low-Dose CT

\begin{tabular}{lc}
\hline Number of ULD CT scans & 118 \\
Number of ULD CT scans with ureteral stones & 50 \\
Stone size, mm & $6.0 \pm 2.9$ \\
Male & $98.0 \%$ \\
Age & 67.1 \\
BMI, $\mathrm{kg} / \mathrm{m}^{2}$ & $30.1 \pm 9.6$ \\
Effective dose, mSv & $1.04 \pm 0.41$ \\
ULD CT scans in patients with previous history & 30 \\
$\quad$ of stones & 21 \\
ULD CT scans in patients with prior & \\
$\quad$ ureteroscopic procedures & \\
Laboratories & \\
$\quad \begin{array}{l}\text { Creatinine, mg/dL } \\
\text { eGFR, mL/min/1.73 m }{ }^{2}\end{array}$ & $1.3 \pm 0.4$ \\
$\quad \begin{array}{l}\text { Number of scans in patients with positive } \\
\quad \text { WBCs }>25 \text { WBCs) on urinalysis }\end{array}$ & $62.8 \pm 18.2$ \\
$\quad \begin{array}{l}\text { Number of scans in patients with positive } \\
\quad \text { leukocyte esterase on urinalysis }\end{array}$ & 8 \\
$\quad \begin{array}{l}\text { Number of scans in patients with positive } \\
\quad \text { nitrites on urinalysis }\end{array}$ & 2 \\
$\quad$
\end{tabular}

$\mathrm{BMI}=$ body mass index; ULD CT $=$ ultra-low-dose $\mathrm{CT}$; $\mathrm{WBC}=$ white blood cells; eGFR = estimated glomerular filtration rate. 
$100.00 \%$

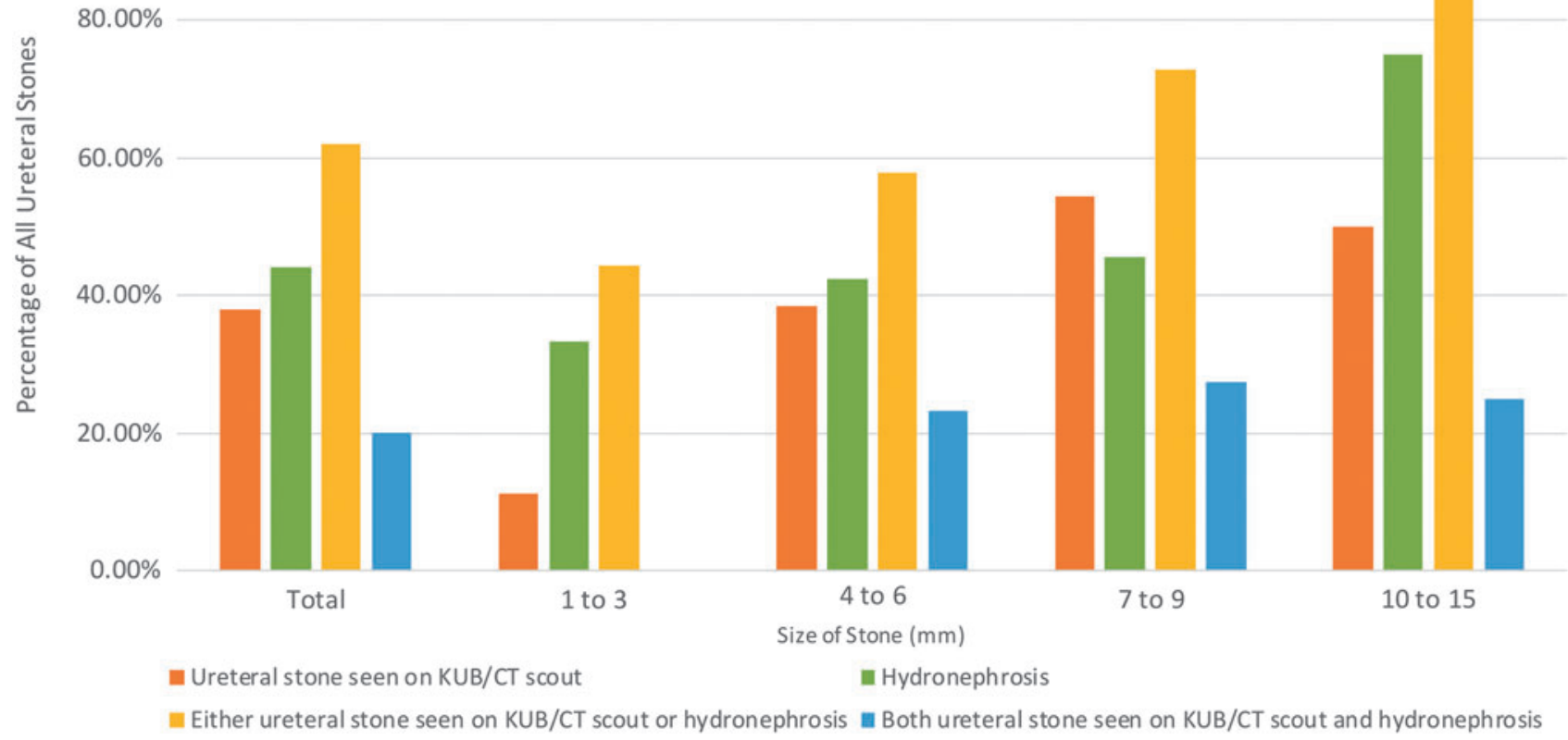

\begin{tabular}{|c|c|c|c|c|c|}
\hline $\begin{array}{l}\text { Ureteral stone size } \\
\qquad(\mathrm{mm})\end{array}$ & Total & 1 to 3 & 4 to 6 & 7 to 9 & 10 to 15 \\
\hline $\begin{array}{c}\text { Ureteral stones seen on } \\
\text { ULD CT }\end{array}$ & $\mathrm{n}=50$ & $n=9$ & $n=26$ & $\mathrm{n}=11$ & $n=4$ \\
\hline $\begin{array}{l}\text { Ureteral stones seen on } \\
\text { KUB/CT scout }\end{array}$ & 19 (38.0\%) & $1(11.1 \%)$ & $10(38.5 \%)$ & $6(54.5 \%)$ & $2(50.0 \%)$ \\
\hline Hydronephrosis & $22(44.0 \%)$ & $3(33.3 \%)$ & $11(42.3 \%)$ & $5(45.5 \%)$ & $3(75.0 \%)$ \\
\hline $\begin{array}{l}\text { Either ureteral stone } \\
\text { seen on KUB/CT scout } \\
\text { or hydronephrosis }\end{array}$ & 31 (62.0\%) & $4(44.4 \%)$ & $15(57.7 \%)$ & $8(72.7 \%)$ & $4(100.0 \%)$ \\
\hline $\begin{array}{l}\text { Both ureteral stone } \\
\text { seen on KUB/CT scout } \\
\text { and hydronephrosis }\end{array}$ & $10(20.0 \%)$ & $0(0.0 \%)$ & $6(23.1 \%)$ & $3(27.3 \%)$ & $1(25.0 \%)$ \\
\hline
\end{tabular}

FIG. 1. Detection of ureteral stones by size.

$12 / 14(85.7 \%)$ of the proximal ureteral stones and 19/36 $(52.8 \%)$ of the distal ureteral stones were detected on either $\mathrm{KUB} / \mathrm{CT}$ scout film or resulted in hydronephrosis (Fig. 2). The mean effective dose per ULD CT was $1.04 \pm 0.41 \mathrm{mSv}$.

Of the ULD CT scans with ureteral stones, 19 (38.0\%) were seen on KUB/CT scout film, 22 (44.0\%) had associated hydronephrosis, $31(62.0 \%)$ were either seen on either
KUB/CT scout film or had hydronephrosis, and only 10 (20.0\%) were both seen on KUB/CT scout film and associated with hydronephrosis (Fig. 1). There was significant association between KUB and ULD CT scout film in detecting ureteral stones by Fisher's exact test $(p=0.004)$, indicating no significant difference between the two modalities in ureteral stone detection.

Table 2. Definitions of Imaging CRiteria

Imaging modality

ULD CT

KUB/CT scout film

KUB/CT scout film or hydronephrosis

KUB/CT scout film and hydronephrosis

\section{Imaging criteria for ureteral stone}

Ureteral stone seen on ULD CT

Ureteral stone seen on plain X-ray KUB or CT scout film

Either ureteral stone seen on KUB/CT scout film or hydronephrosis seen on ULD CT

Both ureteral stone seen on KUB/CT scout film and hydronephrosis seen on ULD CT 


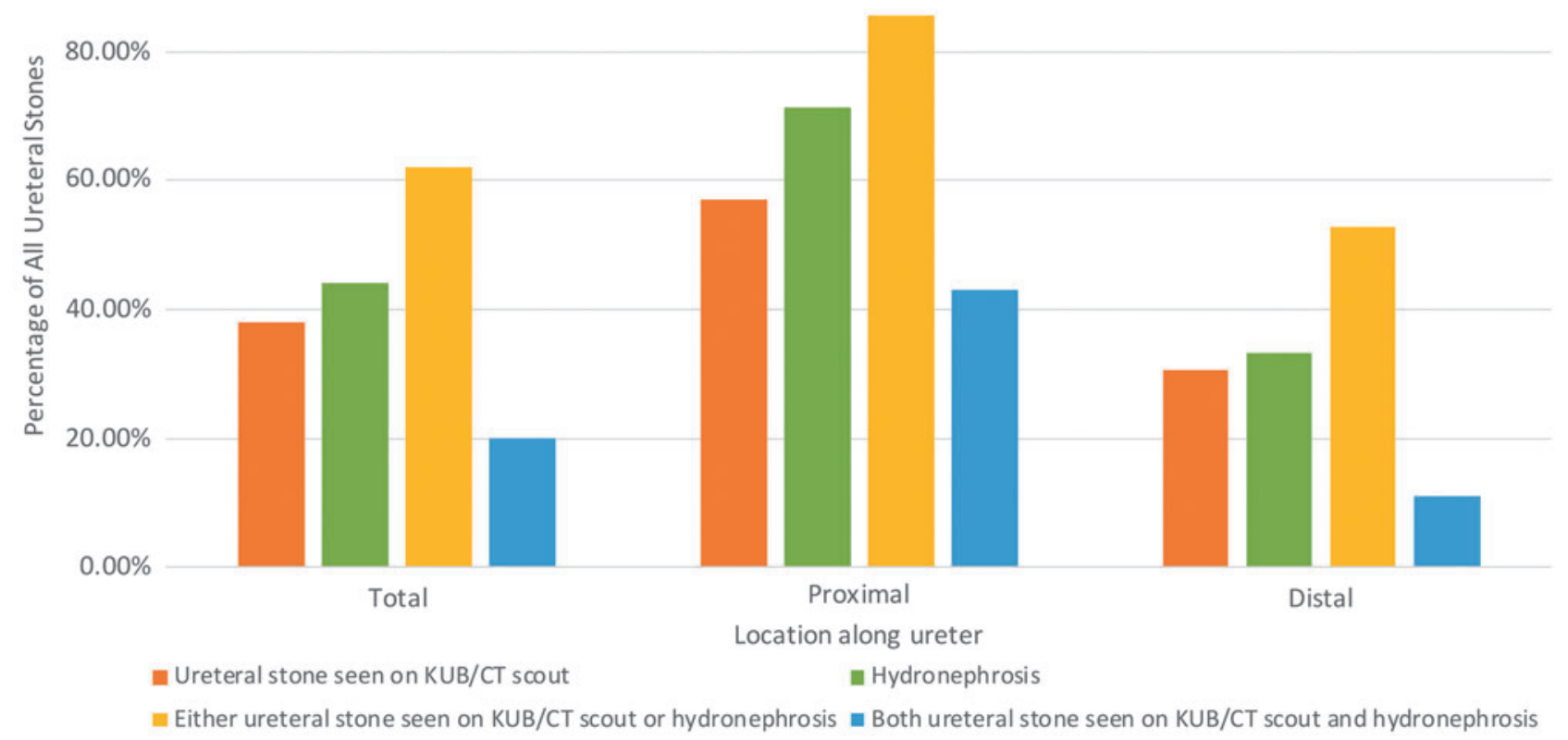

\begin{tabular}{|c|c|c|c|}
\hline Ureteral stone location & Total & Proximal & Distal \\
\hline $\begin{array}{c}\text { Ureteral stones seen on } \\
\text { ULD CT }\end{array}$ & $\mathrm{n}=50$ & $\mathrm{n}=14$ & $11(30.6 \%)$ \\
\hline $\begin{array}{c}\text { Ureteral stones seen on } \\
\text { KUB/CT scout }\end{array}$ & $19(38.0 \%)$ & $10(71.4 \%)$ & $12(33.3 \%)$ \\
\hline Hydronephrosis & $22(44.0 \%)$ & $12(85.7 \%)$ & $19(52.8 \%)$ \\
\hline $\begin{array}{c}\text { Either ureteral stone seen } \\
\text { on KUB/CT scout or } \\
\text { hydronephrosis }\end{array}$ & $10(20.0 \%)$ & $6(42.9 \%)$ & $4(11.1 \%)$ \\
\hline $\begin{array}{c}\text { Both ureteral stone seen } \\
\text { on KUB/CT scout and } \\
\text { hydronephrosis }\end{array}$ & & & \\
\hline
\end{tabular}

FIG. 2. Detection of ureteral stones by location along ureter.

Univariate logistic regression analyses showed that for every $1 \mathrm{~mm}$ increase in ureteral stone size the odds of detection by either KUB/CT scout film or hydronephrosis significantly increased (odds ratio [OR]: 1.40, 95\% confidence interval [CI]: $1.08,1.96)$ (Table 3). Stone location also mattered with distal ureteral stones having a decreased odds of detection compared with proximal ureteral stones (OR: $0.18,95 \%$ CI: 0.03, 0.91), while obesity (BMI $\geq 30$, OR: $0.65,95 \% \mathrm{CI}: 0.20,2.05)$ and presence of stone-related symptoms (OR: 0.72, 95\% CI: 0.17, 3.30) did not impact ureteral stone detection. On multivariable logistic regressions these trends continued for stone size (OR: 1.54, 95\% CI: 1.11, 2.39), though location of stone became nonstatistically significant while still showing a similar trend (OR: 0.20, 95\% CI: 0.03, 1.04).

\section{Discussion}

While significantly reducing the radiation dose compared with conventional CT, our application of ULD CT in the surveillance of ureterolithiasis allowed for the detection of ureteral stones that theoretically would have been missed by renal bladder ultrasound (RBUS) and KUB. In our patient cohort, only $38 \%$ of ureteral stones found by ULD CT were visible on KUB/CT scout film, only $44 \%$ of ureteral stones had hydronephrosis (and thus would likely have been found by RBUS), and $38 \%$ of stones were neither seen on KUB/CT scout film nor associated with hydronephrosis. Hence, stone surveillance strategies reliant on KUB, ultrasound, or a combination could miss anywhere from $38 \%$ to $62 \%$ of ureteral stones. We also found that smaller stones and stones in the distal ureter are more likely to be missed when crosssectional imaging is not employed, due to relative lack of hydronephrosis and obscuring bony structures. Given the possible risk of silent renal obstruction, which may result due to untreated ureteral stones, high sensitivity is critical for both diagnosis and monitoring.

Cumulative radiation doses over time are salient in the setting of genitourinary stone surveillance of patients on 
Table 3. Logistic Regression for Variables That Are Predictive of Being Detected as Ureteral Stone on Plain KUB/CT Scout Film OR Through HydronePHrosis

\begin{tabular}{lcc}
\hline & $\begin{array}{c}\text { Univariable, } \\
\text { OR }(95 \% \text { CI })\end{array}$ & $\begin{array}{c}\text { Multivariable, } \\
\text { adjusted OR } \\
(95 \% \text { CI })\end{array}$ \\
\hline $\begin{array}{l}\text { Stone size (per mm) } \\
\text { Stone location } \\
\quad \text { Proximal }\end{array}$ & $1.40(1.08,1.96)$ & $1.54(1.11,2.39)$ \\
$\quad$ Distal & Ref. & Ref. \\
BMI & $0.18(0.03,0.81)$ & $0.20(0.03,1.04)$ \\
$\quad$ Nonobese & Ref. & Ref. \\
$\quad$ Obese & $0.65(0.20,2.05)$ & $0.40(0.08,1.66)$ \\
$\begin{array}{l}\text { Symptomatic } \\
\text { Asymptomatic }\end{array}$ & Ref. & Ref. \\
$\quad$ Symptomatic & $0.72(0.17,3.30)$ & $0.92(0.17,5.14)$ \\
\hline
\end{tabular}

$\mathrm{CI}=$ confidence interval; $\mathrm{OR}=$ odds ratio.

medical expulsive therapy for ureteral stones. A common practice to reducing radiation exposure includes the use of ultrasound and KUB either independently or in combination, to evaluate for stone expulsion rather than a normal-dose $\mathrm{CT}$ scan. Unfortunately, KUB and RBUS imaging for stones lack anatomic detail and adequate sensitivity, as evidenced by a recent study finding that one in five patients with upper tract renal stones may be counseled inappropriately if discussion is based on ultrasound data alone. ${ }^{20} \mathrm{We}$ found that the average radiation dose for ULD CT is $1.04 \mathrm{mSv}$, which is lower than both the mean effective plain KUB radiation dose of 2.15 $\mathrm{mSv}^{17}$ and the mean low-dose CT radiation dose of $3.0 \mathrm{mSv}{ }^{5}$ It is the experience at our center, and recently corroborated by published data by Kuebker and colleagues that KUB results in a higher than expected radiation dose because in many instances more than one view is required for the whole field to be completely imaged. ${ }^{17}$ Given the higher sensitivity of ULD CT compared with KUB and ultrasound, improved anatomic detail, and low radiation dose, we feel that ULD CT should replace KUB and ultrasound for the surveillance of known ureteral stones in most instances.

Our study is not without limitations. Specifically, hydronephrosis on ULD CT was used as a proxy for hydronephrosis that would be detected on ultrasound. Ultrasound, however, may in fact be able to diagnose a distal ureteral stone at the ureterovesical junction or the proximal ureter independent of hydronephrosis, which might underestimate the sensitivity of ultrasound imaging. As ultrasounds were not done simultaneously on patients in our study, we could not test this theory. In addition, we did not have KUBs available for all patients, though in those patients with both an ULD CT and KUB, there was no difference in stone detection between the scout film findings and KUB. Prior studies on the use of CT scout film as a substitute for plain KUB have shown that $77 \%$ of ureteral stones visible on CT scout film are visible on KUB. ${ }^{21,22}$ In addition, our protocol was restricted to patients with a BMI $\leq 40$, and patients with higher BMI may have differences in required radiation doses for detection of stones and visibility of ureteral stones on ULD CT. As we did not conduct a control imaging study such as low-dose CT or normal-dose CT to look for stones that
ULD CT may have missed, we do not have data on the falsenegative rate of ULD CT. Distal ureteral stones may be mistaken for phleboliths or other calcifications outside the urinary tract even on standard-dose CT. ${ }^{23}$ This is what generally leads to most of the false negatives on $\mathrm{CT}$ and is likely amplified in the ULD setting as resolution is slightly compromised.

In our Veterans Health Administration setting, we have the advantage of working in a closed system with a small group of radiologists. Hence, the time-consuming process of manually protocoling each scan was not a large undertaking. In addition, as the radiation dose is lowered, resolution of the solid organs in the field is decreased, and other abnormal findings on the low-resolution study might be missed though the resolution is adequate for stone detection. An attempt to implement this protocol in a larger practice setting without a collaborative relationship with radiologists may be met with resistance. Despite this, implementation is feasible and would likely improve the current management paradigm for patients with ureteral stones. Finally, our study was done at a single institution, potentially reducing generalizability to other practice settings and as the Veterans Affairs system does not have cost charges, we could not account for cost considerations.

Beyond ureterolithiasis, ULD CT may be of added utility in surveillance of renal stones that are not visible or well characterized on ultrasound or KUB. Future analysis will focus on the application of ULD CT to replace KUB and ultrasound as part of a nephrolithiasis surveillance protocol.

\section{Conclusions}

ULD CT is an effective imaging technique for detecting ureterolithiasis that limits radiation doses to levels comparable with those of a single-view KUB. Our data support the utilization of ULD CT to replace KUB and ultrasound for the surveillance of ureteral stones, especially those that are small or located in the distal ureter.

\section{Author Disclosure Statement}

No competing financial interests exist.

\section{Funding Information}

No funding was received for this article.

\section{References}

1. Scales CD, Smith AC, Hanley JM, Saigal CS. Prevalence of kidney stones in the United States. Eur Urol 2012;62:160165.

2. Ghani KR, Roghmann F, Sammon JD, et al. Emergency department visits in the United States for upper urinary tract stones: Trends in hospitalization and charges. J Urol 2014;191:90-96.

3. Saigal CS, Joyce G, Timilsina AR. Direct and indirect costs of nephrolithiasis in an employed population: Opportunity for disease management? Kidney Int 2005;68:1808-1814.

4. Khan SR, Pearle MS, Robertson WG, et al. Kidney stones. Nat Rev Dis Prim 2016;2:1-22.

5. Fulgham PF, Assimos DG, Pearle MS, Preminger GM. Clinical effectiveness protocols for imaging in the management of ureteral calculous disease: AUA technology assessment. J Urol 2013;189:1203-1213. 
6. Leo M, Langlois B, Pare J, et al. Ultrasound vs. computed tomography for severity of hydronephrosis and its importance in renal colic. West J Emerg Med 2017;18: $559-568$.

7. Rosen CL, Brown DFM, Sagarin MJ, Chang Y, McCabe CJ, Wolfe RE. Ultrasonography by emergency physicians in patients with suspected ureteral colic. J Emerg Med 1998;16:865-870.

8. Kluner C, Hein PA, Gralla O, et al. Does ultra-low-dose CT with a radiation dose equivalent to that of KUB suffice to detect renal and ureteral calculi? J Comput Assist Tomogr 2006;30:44-50.

9. Smith-Bindman R, Aubin C, Bailitz J, et al. Ultrasonography versus computed tomography for suspected nephrolithiasis. N Engl J Med 2014;371:1100-1110.

10. Pai R, Modh R, Lamoureux RH, et al. Image quality and patient-specific organ doses in stone protocol CT: A comparison of traditional CT to low dose CT with iterative reconstruction. Biomed Res Int 2018;2018:5120974.

11. Rob S, Bryant T, Wilson I, Somani BK. Ultra-low-dose, low-dose, and standard-dose CT of the kidney, ureters, and bladder: Is there a difference? Results from a systematic review of the literature. Clin Radiol 2017;72:11-15.

12. Hamm M, Knopfle E, Wartenberg S, Wawroschek F, Weckermann D, Harzmann R. Low dose unenhanced helical computerized tomography for the evaluation of acute flank pain. J Urol 2002;167:1687-1691.

13. Moore CL, Daniels B, Ghita M, et al. Accuracy of reduceddose computed tomography for ureteral stones in emergency department patients. Ann Emerg Med 2015;65:189198.e2.

14. Assimos D, Krambeck A, Miller NL, et al. Surgical management of stones: American Urological Association/Endourological Society Guideline, PART II. J Urol 2016; 196:1161-1169.

15. Yallappa S, Amer T, Jones P, et al. Natural history of conservatively managed ureteral stones: Analysis of 6600 patients. J Endourol 2018;32:371-379.

16. Assimos D, Murad MH, Pearle MS, et al. Surgical management of stones: American Urological Association/Endourological Society Guideline, PART I. J Urol 2016; 196:1153-1160.

17. Kuebker J, Shuman J, Hsi RS, Herrell SD, Miller NL. Radiation from kidney-ureter-bladder radiographs is not trivial. Urology 2019;125:46-49.
18. Tack D, Sourtzis S, Delpierre I, de Maertelaer V, Gevenois PA. Low-dose unenhanced multidetector CT of patients with suspected renal colic. Am J Roentgenol 2003;180:305-311.

19. RStudio Team. RStudio: Integrated Development for R. 2016. www.rstudio.com Accessed August 11, 2019.

20. Ganesan V, De S, Greene D, Cesar F, Torricelli M, Monga M. Accuracy of ultrasonography for renal stone detection and size determination: Is it good enough for management decisions? BJU Int 2017;119:464-469.

21. Ege G, Akman H, Kuzucu K, Yildiz S. Can computed tomography scout radiography replace plain film in the evaluation of patients with acute urinary tract colic? Acta radiol 2004;45:469-473.

22. Johnston R, Lin A, Du J, Mark S. Comparison of kidneyureter-bladder abdominal radiography and computed tomography scout films for identifying renal calculi. BJU Int 2009; 104:670-673.

23. Traubici J, Neitlich JD, Smith RC. Distinguishing pelvic phleboliths from distal ureteral stones on routine unenhanced helical CT: Is there a radiolucent center? Am J Roentgenol 1999;172:13-17.

Address correspondence to: Simon L. Conti, MD, M.Ed Department of Urology Stanford University School of Medicine S287 Grant 300 Pasteur Drive Stanford, CA 94305

E-mail: sconti@stanford.edu

$\begin{aligned} & \text { Abbreviations Used } \\ & \text { ASIR }=\text { adaptive statistical iterative reconstruction } \\ & \mathrm{BMI}=\text { body mass index } \\ & \mathrm{CI}=\text { confidence interval } \\ & \mathrm{CT}=\text { computed tomography } \\ & \mathrm{KUB}=\text { kidney, ureter, and bladder radiograph } \\ & \mathrm{OR}=\text { odds ratio } \\ & \mathrm{RBUS}=\text { renal and bladder ultrasound } \\ & \mathrm{ULD} \mathrm{CT}=\text { ultra-low-dose computed tomography } \\ & \mathrm{WBC}=\text { white blood cells }\end{aligned}$

